\title{
Photodegradation of Polycyclic Aromatic Hydrocarbon Phenanthrene on Soil Surface
}

\author{
Hua Tian \\ College of Geology and Environment \\ Xi'an University of Science and Technology \\ Xi'an, China \\ Tianhua7366@163.com
}

\author{
Zhe Liu \\ College of Geology and Environment \\ Xi'an University of Science and Technology \\ Xi'an, China \\ 664887996@qq.com
}

\begin{abstract}
Photodegradation of Polycyclic Aromatic Hydrocarbons (PAHs) is a main environmental behavior and plays an important role in the fate of PAHs in environment. In order to understand the photodegradation law of Polycyclic Aromatic Hydrocarbons (PAHs) in soil, aeolian sand in blow-sand region of Northern Shaanxi was chosen as typical soil and phenanthrene (Phe) as typical pollutant of PAHs. Photodegradation law of Phe on soil surface was simulated. In addition, effects of initial Phe concentration, light intensity, time and $\mathrm{pH}$ on photodegradation of Phe in aeolian sand was assessed. Results show that the photodegradation of Phe followed pseudo first-order kinetics. Photodegradation rate constant of Phe increased with the increasing of irradiation intensity which the order was as followed: $125 \mathrm{~W}>100 \mathrm{~W}>75 \mathrm{~W}>50 \mathrm{~W}$. Irradiation intensity enhances photodegradation of PAHs on soil surfaces. Photodegradation half-life of Phe ranged from 2.474 hours to 3.095 hours under different irradiation intensity. Degradation rate increases as $\mathrm{pH}$ increase and photodegradation is more rapid in alkaline medium. Photodegradation rate is the lowest as initial concentration is the largest.
\end{abstract}

Keywords-photodegradation; Phenanthrene(Phe); aeolian sand; photodegradation kinetics; influence factors

\section{INTRODUCTION}

Polycyclic aromatic hydrocarbons (PAHs) is a kind of typical persistent organic pollutants, mainly derived from natural and anthropogenic sources, generated during the incomplete combustion of solid and liquid fuels or derived from industrial activities. PAHs are known to be widespread pollutants which are teratogenic, carcinogenic and mutagenic toxic ${ }^{[1-4]}$. In US EPA priority pollutants list, there are 16 PAHs included ${ }^{[5]}$. Thus PAHs are ubiquitous pollutants that occur in natural phase such as soil, sediment, water and air, and are harmful to environment and human health. Because of low solubility, PAHs are easy to go into the water phase. Most of PAHs can be adsorbed on soil in environment and only few can enter into the water body. So soil is a very important focus place and medium bearing PAHs. Migration of PAHs in soil is slow and it mainly focused on the soil surface layer ${ }^{[6]}$. The main processes which can successfully remove and eliminate the organic pollutants in environment mainly include: microbiological transformation and degradation, volatilization, photodegradation and so on ${ }^{[7]}$. Among these processes, photodegradation is one of the major abiotic transformation processes for PAHs in the environment ${ }^{[8]}$. Thus it is importance to investigate photodegradation of PAHs in environment. It can help us to develope efficient and economical degradation technologies for PAHs.

Over the past few years, much work has been done in characterizing photolytic reactions of PAHs in aqueous ${ }^{[9-11]}$. On the other hand, Photodegradation of organic matter in soil is influenced by various environmental factors which include irradiation intensity, soil composition and the nature of physical and chemical properties, organic matter and so on. The photodegradation of organic pollutants in the soil depends not only on the degradation rate, but also on the soil thickness, moisture, diffusion and migation of pollutants and other processes. Soil can effectively block light and reduce light penetration ${ }^{[12]}$. When other conditions are same, the photodegradation of organic matter in soil was accelerated with different $\mathrm{pH}$ and depth in soil ${ }^{[13]}$. The photochemical degradation of PAHs in soil usually occured in the soil/air interface which its thickness less than $1 \mathrm{~mm}$. Direct photodegradation process generally occured mainly in soil surface layer of $0.2-0.4 \mathrm{~mm}$. Thickness about $0.5 \mathrm{~mm}$ can block the $95 \%$ incident light. Only little can penetrate through the $1.5 \mathrm{~mm}$ or deeper in soil layer ${ }^{[14]}$. The photodegradation in a certain thickness of soil followed pseudo-first-order kinetics ${ }^{[15-16]}$. But sometimes the secondary rate constant or Hoerl function can better reflect the relationship between various factors $^{[17-18]}$. Humus effect on the photodegradation of DDT was accord with Langmuir-Hinshel Wood model ${ }^{[19]}$.

Recent studies have focused on the photodegradation of PAHs in the soils. However, comparatively little research effort has been directed at examining the photodegradation of Phenanthrene(Phe) on eolian sand, and the fate of Phe adsorbed on eolian sand surfaces has not been fully explored.

The purpose of this study therefore was to investigate photodegradation of PAHs on aeolian sand surfaces under irradiation. Phenanthrene was chosen as a representative compounds for PAHs. Halogen tungsten lamp was use to simulate the natural light. Using photochemical test on soil surface, photodegradation kinetic model and influence factors were discussed. Rresult is of great importance to investigate the transport and transformation of PAHs. 


\section{MATERIALS AND METHODS}

\section{A. Chemicals and apparatus}

Phenanthrene was purchased from Aladdin Industrial Corporation and the purity was 95\% used without further purification. Methanol (Analytical Reagent, AR) was purchased from Beijing Chemical Works, China. Anhydrous calcium chloride (AR) was purchased from Tianjin Fengchuan Chemical Reagent Science And Technology Co., Ltd. The type of constant temperature drying oven was DHG-9070A and was purchased from Shanghai Yiheng Technical Co., Ltd. Millie-Q water purification machine was Gradient A10 from Millipore Corporation, USA. Ultraviolet spectrophotometer was purchased from Purkinje General and the type of $\mathrm{pH}$ meter was JSM-6460LV. Oscillator, ultrasonic bath and centrifuge was also needed in the experiment. Tungsten halogen lamps were used as the irradiation sources which the wattages were 50, 75, 100 and 125 respectively.

\section{B. Soil samples}

Surface soil samples were collected from the blowsand region of the Northern Shaanxi. The soil was the typical eolian sand in this area. Impurities were removed from the soil samples and air dried. Then the soil samples were passed through a 1-mm sieve and stored in a dark chamber before use. After autoclaving, the soil samples were mixed with methanol solution. Then let the methanol volatile completely.

\section{Photodegradation experiments}

In all photodegradation experiments, different replicates of $25 \mathrm{~g}$ polluted soil samples were evenly spread on Petri dishes which diameter was $9 \mathrm{~cm}$ and located in a chamber. The soil thickness was $2 \mathrm{~cm}$. Put the Petri dish with polluted soil samples under the irradiation of tungsten halogen lamp which the distance was $30 \mathrm{~cm}$. Light-proof Petri dishe also containing $25 \mathrm{~g}$ of uniformly spread soil samples was used as controls for the measurement of non-photodegradation PAHs loss for all experimental treatments. Temperature within the chamber was held constant at $25^{\circ} \mathrm{C}$ throughout all experiments. Soil samples were taken at 1 hour intervals from the Petri dishes exposed to light.

The effect of irradiation intensity on the photodegradation was investigated by conducting experiments. Five different levels of irradiation intensity were employed in the range of no light, 50W, 75W, $100 \mathrm{~W}$ and $125 \mathrm{~W}$. The effect of time about $0 \mathrm{~h}, 1 \mathrm{~h}, 2 \mathrm{~h}, 3 \mathrm{~h}, 4 \mathrm{~h}$ and $5 \mathrm{~h}$ on the degradation of PAHs was also examined. The $\mathrm{pH}$ of soil samples on the Petri dishes were adjusted from 3 to 11. Initial Phe concentration in the soil samples were $60 \mathrm{mg} / \mathrm{g}, \quad 90 \mathrm{mg} / \mathrm{g}, 120 \mathrm{mg} / \mathrm{g}, 150 \mathrm{mg} / \mathrm{g}, 180 \mathrm{mg} / \mathrm{g}$, respectively.

\section{Analytical methods}

Concentrations of PAHs in the irradiated and nonirradiated soil samples were obtained by the following method: the soil samples were transferred into $50 \mathrm{~mL}$ centrifugal tubes and added with $10 \mathrm{~mL}$ methanol. The centrifugal tubes were covered by tinfoil to prevent the light irrigation. The mixtures were extracted for 30min in an ultrasonic bath extractor at the controlled temperature below $10{ }^{\circ} \mathrm{C}$. After extraction, the mixtures were then centrifuged at $4000 \mathrm{r} / \mathrm{min}$ for $5 \mathrm{~min}$ to separate the supernatant from the soil samples. The same extraction process was repeated three times. The effluent samples were collected and diluted to appropriate concentration for later analysis. Quantification of PAHs in methanol solutions was conducted using an ultraviolet spectrophotometer with a wavelength set at $250 \mathrm{~nm}$.

\section{RESULTS AND DISCUSSION}

\section{A. Physical and chemical properties of soil}

Mechanical composition of aeolian sand was fine. Particle size between 0.074 to $0.250 \mathrm{~mm}$ was more than $90 \%$, more than $0.25 \mathrm{~mm}$ was only $0.1 \%$ and less than $0.074 \mathrm{~mm}$ was less than $9 \%$. The coefficient of nonuniformity was about 1.35. Mineral element of the experimental soil samples was analyzed by energy spectrum analysis and the main chemical component was $\mathrm{SiO}_{2}$.

\section{B. Law of photodegradation}

Degradation law of Phe measured at 1 hour intervals were shown in fig.1. It was observed that degradation of Phe under dark condition mainly occurred in the first hour which was achieved $7.18 \%$. Then the concentration of PAHs was not altered obviously and degradation rate in five hours was only $9.97 \%$. On the contrary, concentration of Phe decreased when irradiation time prolonged. Photodegradation rate was fast in the first four hours and then became slow. Residual rate of Phe was 36.84\% after four hour irradiation. It showed that photochemical reaction rate was faster in the early irradiation with more molecules of PAHs on soil surface. Phe concentration on soil surface decreased and photoreaction rate also decreased. Hence, the results indicated that the degradation degree of PAHs on soil surfaces was related to irradiation on soil surface.

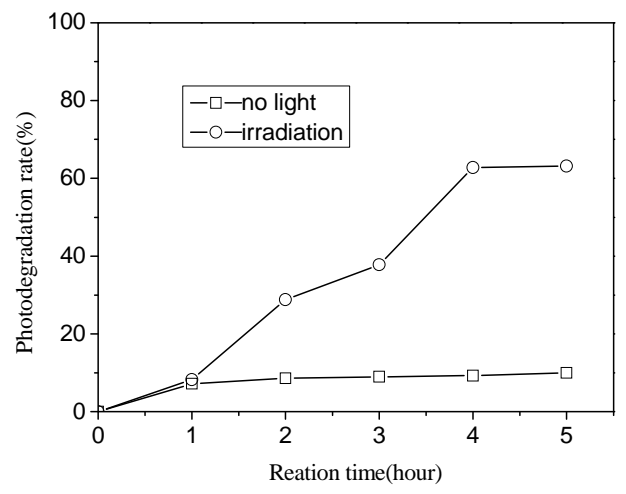

Figure 1. Photodegradation curves of Phe

\section{Irradation intensity effect}

It is well established that light intensity is an important factor which affects photodegradation of organics. Photodegradation rate increases with the irradiation intensity enhanced ${ }^{[19]}$. Eeffect of irradiation 
intensity on Phe degradation was investigated in 5 hours and photodegradation rate of Phe in eolian sand was shown in Fig.2.

It was observed that intensity increased from $50 \mathrm{~W}$ to $125 \mathrm{~W}$, the degradation rate of Phe increased from $63.16 \%$ to $73.55 \%$ after 5 h reaction. The degradation rate of Phe under same conditions was in the sequence $125 \mathrm{~W}>100 \mathrm{~W}>75 \mathrm{~W}>50 \mathrm{~W}>$ no irradiation.

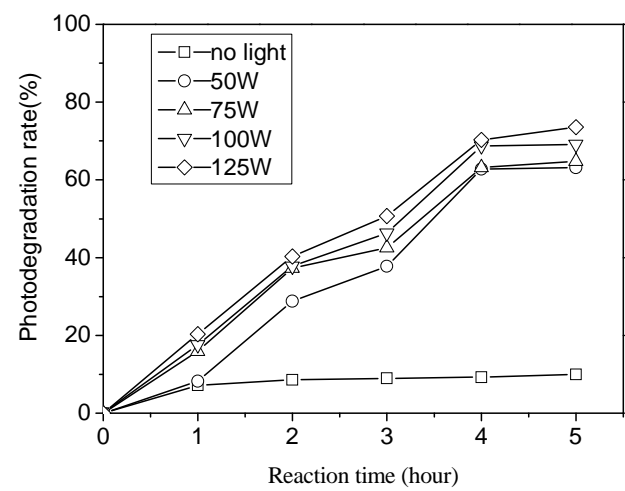

Figure 2. Effect of irradiation intensity

The results indicated that the irradiation intensity enhanced the degradation of the Phe on soil surfaces. Theoretically, at a given depth, the rate of photoreaction was assumed to obey a first-order rate law ${ }^{[5,16]}$. On the other hand, greater irradiation intensity provided more energy for Phe particles, and the reaction rate therefore increased with increased irradiation intensity ${ }^{[20]}$.

\section{D. $p H$ effect}

Soil $\mathrm{pH}$ is an important influencing factor and appropriate $\mathrm{pH}$ could speed up the photodegradation of organics $^{[21]}$. Effect of $\mathrm{pH}$ was showed in Fig.3. Results indicated that $\mathrm{pH}$ had less effect on photodegradation of Phe. With $\mathrm{pH}$ increases, the degradation rate slightly ascended. This is because acid and alkali could not play a role in the dry sand like in solution where can provides hydrogen ions and hydroxyl.

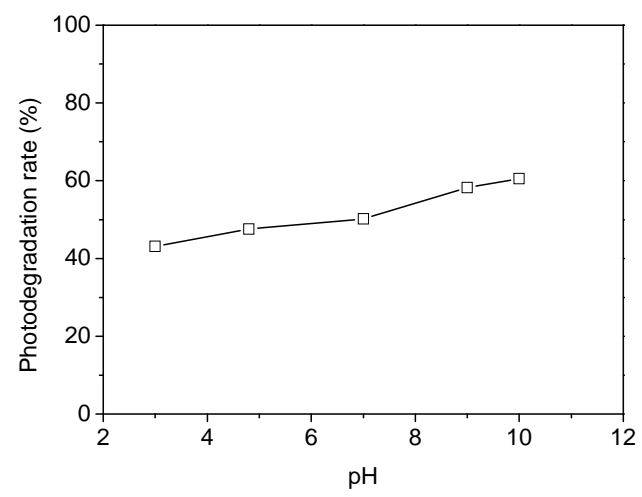

Figure 3. Effect of $\mathrm{pH}$

\section{E. Initial concentration effect}

Figure 4 showed the effect of initial concentration on the photodegradation of Phe under $125 \mathrm{~W}$ with different initial concentration. Content of Phe decreased after 5 hours and degradation percentages were 64.35\%, 59.87\%, $55.79 \%, 52.67 \%$ and $49.03 \%$ respectively. Result showed that photodegradation rate was obviously influenced by initial concentration. Higher concentration of Phe makes the solution less permeable to light irradiation under the same irradiation intensity. It can also be confirmed that PAHs should be effectively photodegraded on soil surface.

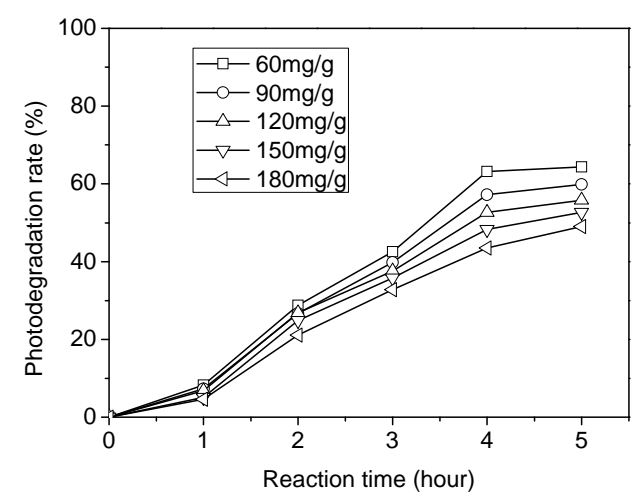

Figure 4. Effect of initial concentration

\section{F. Photodegradation kinetics}

Concentration gradient of organic in soil vertical depends on the photodegradation, which follows the pseudo first-order rate equation ${ }^{[15]}$. Most direct photodegradation of organic pollutants in homogeneous system obeyed the pseudo first-order rate equation ${ }^{[5]}$. Equation and parameters were fitted with the photodegradation data of Phe in the soil under different irradiation intensity. Results were listed in Table 1.

The fitting result showed that photodegradation of Phe followed the pseudo-first order kinetics. The pseudofirst order rate constant was increased as the irradiation intensity increased. The maximum was $0.2806 \mathrm{~h}$ which the irradiation intensity was $125 \mathrm{~W}$. Half-life was shortened when irradiation intensity was enhanced. The minimum was $2.474 \mathrm{~h}$ which irradiation intensity was $125 \mathrm{~W}$.

\section{CONCLUSION}

Results of the present work suggested that photodegradation was a successful way to remediate PAHs-contaminated soils. Degradation rate of Phe on aeolian sand increased with time prolonged. The process follows pseudo-first order kinetics. It was significantly influenced by irradiation intensity and initial concentration.

\section{ACKNOWLEDGEMENTS}

This work was supported by the National Natural Science Foundation of China (Grant No. 41202176), Scientific Research Program Funded by Shaanxi Provincial Education Department (Program No. 12JK0645), Open Fund of Chang'an University (No. 2013G1502034) and Doctor Research Fund of Xi'an University of Science and Technology (2012QDJ018). 
TABLE 1. PHOTODETRADATIN KINETIC PARAMETERS OF PHE UNDER DIFFERENT IRRADIATION INTENSITY

\begin{tabular}{|ccccc|}
\hline $\begin{array}{c}\text { Irradiation } \\
\text { Intensity } \\
/ W\end{array}$ & Pseudo first-order kinetics & $\begin{array}{c}\text { Rate constant } \\
/ \mathrm{h}\end{array}$ & $\begin{array}{c}\text { Half-life } \\
/ \mathrm{h}\end{array}$ & $\begin{array}{c}\text { Correlation coefficient } \\
R\end{array}$ \\
\hline 55 & $C_{\mathrm{t}}=2.617 e^{-0.2239 t}$ & 0.2239 & 3.095 & 0.9696 \\
75 & $C_{\mathrm{t}}=2.512 e^{-0.2242 t}$ & 0.2242 & 3.056 & 0.9829 \\
100 & $C_{\mathrm{t}}=2.589 e^{-0.2553 t}$ & 0.2553 & 2.717 & 0.9792 \\
125 & $C_{\mathrm{t}}=2.603 e^{-0.2806 t}$ & 0.2806 & 2.474 & 0.9895 \\
\hline
\end{tabular}

\section{REFERENCES}

[1] Liu L B, Liu Y, Lin J M, Tang N, Hayakawa K, Maeda T. Development of analytical methods for polycyclic aromatic hydrocarbons (PAHs) in airborne particulates: A review. Chinese Journal of Environmental Science, 2007, 19(1):1-11.

[2] Ma J Y, Zhou Z. "Soil pollution by polycyclic aromatic hydrocarbons: A comparison of two Chinese cities", Chinese Journal of Environmental Science, 2011, 23(9): 1518-1523.

[3] Na G S, Liu C Y, Wang Z, et al. "Distribution and characteristic of PAHs in snow of Fildes Peninsula", Chinese Journal of Environmental Science, 2011, 23(9): 1445-1451.

[4] Sun R, Jin J H, Sun G D, et al. "Screening and degrading characteristics and community structure of a high molecular weight polycyclic aromatic hydrocarbon degrading bacterial consortium from contaminated soil”, Chinese Journal of Environmental Science, 2010, 22(10): 1576-1585.

[5] Xu C B, Dong D B, Meng X L, et al. "Photolysis of polycyclic aromatic hydrocarbons on soil surfaces under UV irradiation", Chinese Journal of Environmental Science, 2013, 25(3):569-575.

[6] Yao L L, Zhang C X, Li J L, et al. "Vertical distribution and possible sources of polycyclic aromatic hydrocarbon in sewage area soil”, Chinese Journal of Environmental Science, 2013, 34(4): 1553-1560

[7] Liu P Y, Tian L, Chen Y J. "Photodegradation of permethrin on the sand surface”, Journal of Hebei University (Natural Science Edition), 2014, 34(2): 160-165

[8] Zhang L H, Li P J, Li X M, et al. "Research advance in photodegradation of organic pollutants in surface soil”, Chinese Journal of Ecology, 2006, 25(3): 318-322.

[9] Lehto K M, Vuorimaa E, Lemmetyinen $H$. "Photolysis of polycyclic aromatic hydrocarbons (PAHs) in dilute aqueous solutions detected by fluorescence”, Journal of Photochemistry and Photobiology A: Chemistry, 2000, 136(1-2): 53-60.

[10] Sanches S, Leit-ao C, Penetra A, et al. "Direct photolysis of polycyclic aromatic hydrocarbons in drinking water sources", Journal of Hazardous Materials, 2011, 192(3): 1458-1465.
[11] Leite N F, Peralta-Zamora P, Grassi M T. "Distribution and origin of polycyclic aromatic hydrocarbons in surface sediments from an urban river basin at the Metropolitan Region of Curitiba, Brazil”, Journal of Environmental Sciences, 2011, 23(6): 904-911.

[12] Miller G C, Herbert V R, Miille M J, et al. "Photolysis of octachlorodibenzo-p-dioxin on soils: production of 2,3,7,8-TCDD”, Chemosphere, 1989, 18(1-6): 1265-1274.

[13] Zheng H H, Ye C M. "Photodegradatin of acetochlor and butachlor in soil”, Environmental Chemistry, 2002, 21(2):117-122.

[14] Frank M P, Graebing P G, Chib J S. "Effect of soil moisture and sample depth on pesticide photolysis”, Journal of Agricultural and Food Chemistry, 2002, 50: 2607-2614.

[15] Balmer M E, Goss K U, Schwarzenbach R P. “ Photolytic transformation of organic pollutants on soil surfaces-an experimental approach”, Environmental Science and Technology, 2000, 34(7): 1240-1246.

[16] Si Y B, Yue Y D, Zhou D M, Chen H M. "Photodegradation of pesticide in soil”, Rural Eco-Environment, 2002, 18(4): 56-59.

[17] Emmelin C, Guittonneau S, Lamartine R, et al. Photodegradation of pesticides on adsorbed phases. Photodegradation of carbetamid [J].Chemosphere, 1993, 27:757-763.

[18] Romero E, Dios G, Mingorance M D, et al. Photodegradation of mecoprop and dichloprop on dry-moist and amended soil surfaces exposed to sunlight [J].Chemosphere, 1998, 37(3): 577-589.

[19] Zhang L H, Li P J, Li X M, Gong Z Q, Zhang H R, Xu H X. Research advance in photodegradation of organic pollutants in surface soil. Chinese Journal of Ecology 2006, 25(3): 318-322.

[20] Li Y X, Duan X Y, Li X G, et al. "Photodegradation of nonylphenol by simulated sunlight”, Marine Pollution Bulletin, 2013, 66: 47-52.

[21] Metsarinne S, Tuhkanen T, Aksela R. "Photodegradation of Ethylenediaminetetraacetic Acid (EDTA) and Ethylenediamine Disuccinic Acid (EDDS) within Natural UV Radiation Range. ”, Chemosphere, 2001, 48: 949-955. 